\title{
Existe-t-il des métaux à deux dimensions ?
}

Geneviève Fleury ${ }^{(1)}$ (Genevieve.Fleury@unige.ch) et Xavier Waintal (xavier.waintal@cea.fr) Service de physique de l'état condensé, DSM/IRAMIS, CEA/Salay, 91191 Gif-sur-Yvette Cedex (1) Lauréate du prix de l'exposé de thèse au Congrès général 2009 de la SFP.

Les propriétés de conduction des systèmes bidimensionnels d'électrons que l'on trouve par exemple dans les transistors de nos ordinateurs,

font depuis plus de quinze ans l'objet d'un débat qui divise les physiciens de la matière condensée. Alors que l'on pensait qu'en dimension deux il ne pouvait exister que des isolants à cause du désordre inévitable dans les échantillons, des expériences, menées depuis 1994 dans des transistors de nouvelle génération, ont montré d'étranges comportements métalliques.

Par une approche numérique, nous montrons que les interactions coulombiennes entre électrons, considérables dans ces nouveaux échantillons, sont à l'origine de ces observations expérimentales.

* « Il faut s'abaisser à effectuer des simulations numériques pour résoudre même les plus simples des questions concernant [la localisation]."

\section{Les concepts de métal et d'isolant}

En physique de la matière condensée, les matériaux sont classés en deux catégories, selon leurs propriétés de conduction électrique : les métaux et les isolants. Dans les métaux, comme le cuivre ou l'or, des électrons peuvent conduire le courant à température nulle, si l'on applique au matériau un champ électrique. En revanche, en l'absence d'excitations thermiques, aucun courant ne peut traverser un isolant (diamant, PVC...). Ainsi, les métaux se distinguent des isolants par le fait qu'à la limite théorique de température nulle, leur résistivité électrique est finie (par opposition à infinie). Attention toutefois : cette définition théorique peut parfois paraître contradictoire avec l'idée commune que l'on se fait d'un métal. En effet, certains isolants au sens strict conduisent très bien le courant aux températures usuelles (de sorte qu'on les considère comme des métaux pour toute considération pratique) ; mais, à très basse température, ils s'arrêtent brusquement de conduire. Une raison à ce phénomène, d'origine quantique, est la présence de désordre dans le matériau, bloquant le passage des ondes électroniques (voir encadré, p. 9). C'est à celle-ci que nous allons nous intéresser dans la suite de cet article.

Avant d'aller plus loin, précisons que pour sonder en pratique le caractère métallique ou isolant d'un matériau, on mesure la dépendance en température de sa résistivité. Si celle-ci augmente lorsque la température diminue, on dit que le matériau présente un comportement isolant, puisque l'on peut alors s'attendre à une résistivité infinie à température nulle (les semi-conducteurs classiques, comme le silicium, rentrent dans cette catégorie). À l'inverse, si la résistivité diminue au cours du refroidissement on parle de comportement métallique.

\section{Dans un espace bidimensionnel}

La question posée dans cet article concerne le cas particulier de la dimension deux : est-ce que des électrons confinés dans un plan peuvent conduire le courant à température nulle en présence de désordre? La question n'est pas seulement théorique car, depuis plusieurs décennies, les physiciens de la matière condensée sont capables de fabriquer de tels systèmes. On peut citer, par exemple, les transistors Si-MOSFETs (Silicium metaloxide-semiconductor field-effect transistor), composants de base des microprocesseurs de nos ordinateurs (fig. 1), ou encore les

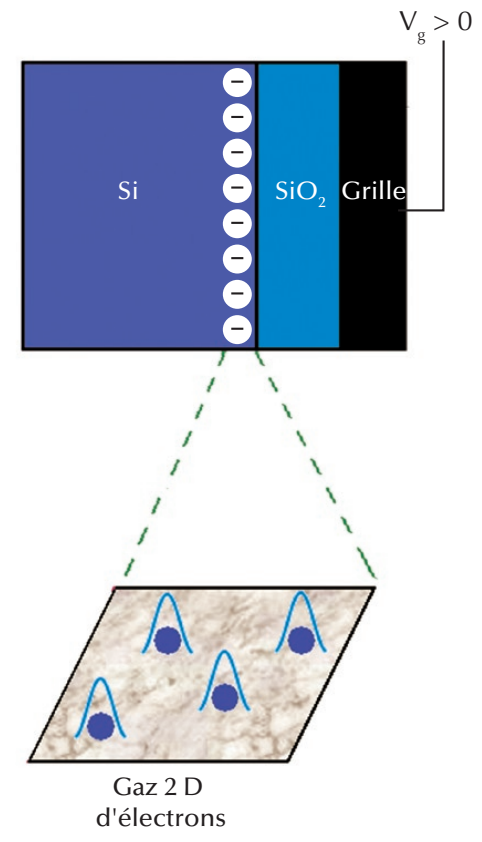

1. Schéma de principe d'un Si-MOSFET. Les électrons du silicium (Si) sont attirés vers la « droite » par l'application d'une tension positive $V_{g}$ sur la grille métallique, mais se retrouvent bloqués par loxyde de silicium (SiO2) isolant.

À l'interface $\mathrm{Si} / \mathrm{SiO}_{2}$ se forme ainsi un système bidimensionnel d'électrons, localisés (fonction d'onde en bleu clair) par la présence inévitable d'un potentiel désordonné. Leur densité peut être modifiée en variant la tension sur la grille. 
One has to resort to the indignity of numerical simulations to settle even the simplest questions about [localization].*, Philip Warren Anderson, prix Nobel de physique 1977

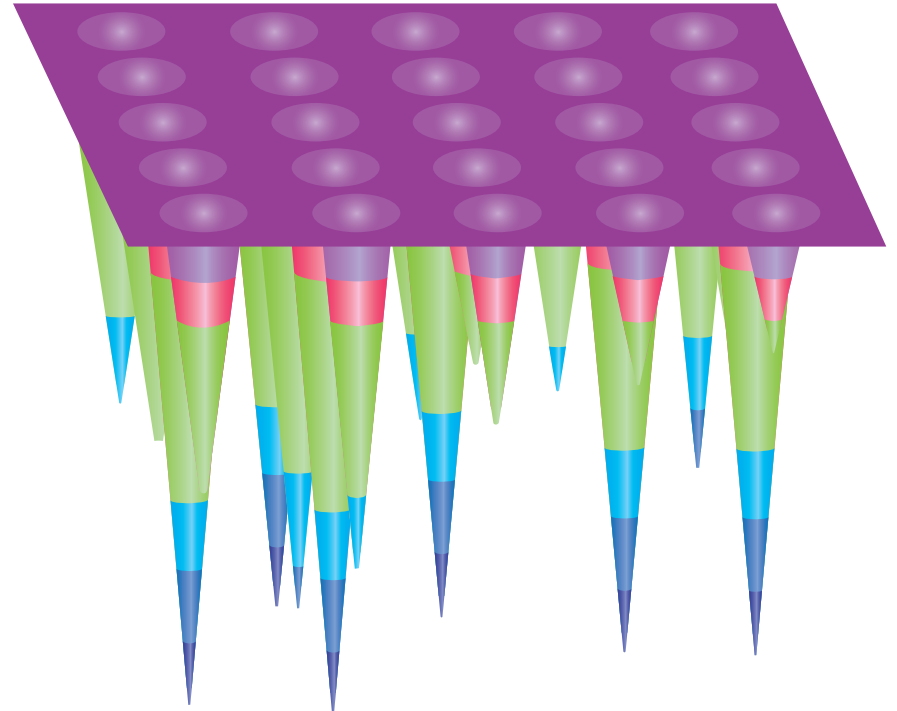

Réseau cristallin bidimensionnel avec une distribution désordonnée de puits de potentiel sur chaque site (inspiré de A. Lagendijk et al., Physics Today 62, nº (2009) 24). hétérostructures semi-conductrices que l'on retrouve dans nos téléphones portables. Dans ces dispositifs, l'idée est de piéger les électrons dans un puits de potentiel de très faible épaisseur à l'interface entre deux semiconducteurs (ou entre un semi-conducteur et un isolant), de sorte que le mouvement des électrons devienne strictement bidimensionnel dans le plan de l'interface. En pratique, des impuretés et des défauts répartis aléatoirement dans le matériau sont à l'origine d'un potentiel désordonné dans lequel évoluent les électrons, couplés entre eux par l'interaction coulombienne.

\section{Dogme des années 1980 : pas de métal à $2 \mathrm{D}$}

Depuis les travaux de P. Anderson [1], il est connu que le désordre peut conduire à la localisation des électrons et ainsi inhiber la conduction électrique du système (voir encadré). Plus précisément, la théorie d'échelle de la localisation ${ }^{(1)}$, élaborée en 1979, prédit qu'à deux dimensions un désordre infinitésimal est suffisant pour localiser les électrons, du moins tant que l'on néglige leurs interactions coulombiennes [2]. C'est ainsi que la communauté s'est convaincue qu'un système bidimensionnel d'électrons indépendants (i.e. sans interaction) doit nécessairement être isolant. Dans certaines limites extrêmes, les interactions ont pu être prises en compte et, à chaque fois, il s'avérait qu'elles n'aidaient pas au transport, bien au contraire [2]. À la fin des années 80, fort de ces arguments théoriques, il était ainsi admis qu'il ne pouvait exister d'état métallique à deux dimensions. Les expériences de l'époque semblaient en outre confirmer ce paradigme puisque seuls des comportements isolants, montrant une divergence de la résistivité à basse température, étaient observés.

\section{Révolution de 1994 : observation de comportements métalliques à 2D}

Le débat sur la question de l'existence de métaux à deux dimensions a été relancé en 1994 par des expériences réalisées par S. V. Kravchenko et ses collaborateurs, dans des Si-MOSFETs de nouvelle génération [3]. En effet, contre toute attente, des comportements métalliques ont été observés dans ces nouveaux échantillons (fig. 2) : au lieu de diverger lorsque la température est diminuée, la résistivité du système chute d'un ordre de grandeur sur une plage de température allant de quelques kelvins à quelques dizaines de millikelvins, laissant ainsi présager une résistivité finie à température nulle (i.e. un état métallique). À l'annonce de ce résultat, apparemment incompatible avec les arguments physiques diffusés depuis plusieurs décennies, la communauté est d'abord restée sceptique. Puis, après confirmation par différentes équipes, l'incrédulité fit place à la polémique : en quelques années, des centaines de publications parurent sur le sujet, les opinions s'affrontèrent lors de multiples conférences, et des modèles théoriques, plus ou moins saugrenus, affluèrent de toutes parts [4]. Quinze ans après, aucun consensus n'a encore été trouvé quant à l'origine de ces comportements métalliques et leur persistance ou non à plus basse température.

De la littérature expérimentale se dégagent toutefois plusieurs observations majeures.

Premièrement, les comportements métalliques ont tous été constatés à basse densité électronique $\left(\sim 10^{11}\right.$ électrons $\left./ \mathrm{cm}^{2}\right)$, dans un régime où les interactions coulombiennes sont trop importantes pour être négligées (voir encadré). En effet, le paramètre $\mathrm{r}_{\mathrm{s}}$ caractérisant l'importance des interactions dans le système, a pu atteindre dans les nouveaux échantillons "métalliques " des valeurs de l'ordre de 10 (voire beaucoup plus), alors que dans les expériences similaires des années 80 , où la densité d'électrons était typiquement dix fois plus élevée, il ne dépassait pas 3 ou 4 . Il se pourrait donc bien que la clé du mystère des comportements métalliques réside dans les interactions électron-électron et leur effet sur la localisation d'Anderson.

Une autre observation est le rôle essentiel du degré de liberté interne de spin (up ou down) des électrons. Il a en effet été montré que si l'on polarise tous les électrons, en

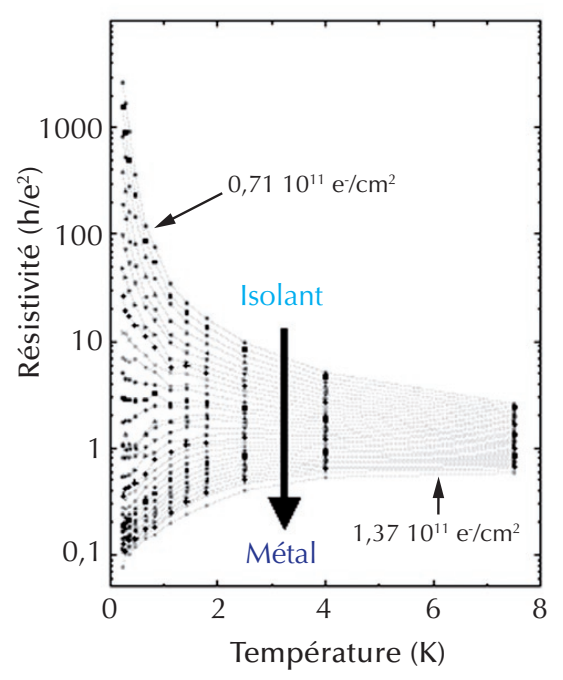

2. Données expérimentales de résistivité d'un système bidimensionnel d'électrons formé dans un Si-MOSFET (en unité du quantum de résistance $\mathrm{h} / \mathrm{e}^{2}$ ) en fonction de la température (en kelvin), pour différentes densités électroniques allant de 0,71 à 1,37 (x $10^{11}$ électrons $/ \mathrm{cm}^{2}$ ) [9]. En augmentant la densité (flèche), on passe d'un comportement isolant (en haut) à un comportement métallique (en bas). 


\section{\>}

appliquant un champ magnétique Zeeman de sorte qu'ils soient tous up, par exemple, les comportements métalliques sont supprimés et l'on retrouve un comportement isolant.

Enfin, le degré de liberté interne de "vallée " des électrons joue aussi un rôle. Il correspond à la possibilité pour chaque électron d'occuper plusieurs puits de potentiel de même énergie, centrés autour de vecteurs d'onde différents. Pour simplifier, on pourra voir ce degré de liberté de vallée comme une couleur (disons bleu ou vert) associée à chaque électron. Dans les Si-MOSFETs où il y a autant d'électrons bleus que verts, les comportements métalliques sont beaucoup plus importants que dans les hétérostructures GaAs/AlGaAs où tous les électrons sont de la même couleur.

\section{Une approche numérique lourde, mais efficace}

L'analyse des données expérimentales montre que la compréhension des comportements métalliques passe par l'étude du régime quantique où désordre et interactions sont tous deux importants. Or ce régime résiste à toute approche analytique. Nous avons ainsi mis au point une méthode numérique pour l'explorer [5]. Partant d'un modèle simple, mais réaliste, contenant comme ingrédients le désordre, les interactions coulombiennes, le spin électronique et le degré de liberté de vallée, on calcule à température nulle l'état fondamental du système bidimensionnel d'électrons, à l'aide d'une méthode stochastique, dite Monte Carlo Quantique $^{(2)}$. Le degré de localisation du système est ensuite "mesuré " via la conductance de Thouless ${ }^{(3)}$. Cette conductance n'est pas directement celle mesurée dans les expériences décrites ci-dessus ; mais elle est sensible aux propriétés de localisation du système électronique et, en outre, elle présente l'énorme avantage d'être facilement calculable par notre méthode numérique. La dernière étape consiste enfin à rechercher la loi d'échelle reliant nos données de conductance à la taille du système, de façon à extraire la limite thermodynamique (affranchie des effets de taille finie).

En pratique, pour effectuer ces simulations numériques complexes, nous avons bénéficié des supercalculateurs du CCRT (logés au CEA/Bruyères-le-Châtel), permettant de calculer sur plusieurs centaines de processeurs en parallèle. Au total, près d'un million d'heures de calcul ont ainsi été dépensées pour mener à bien notre projet !

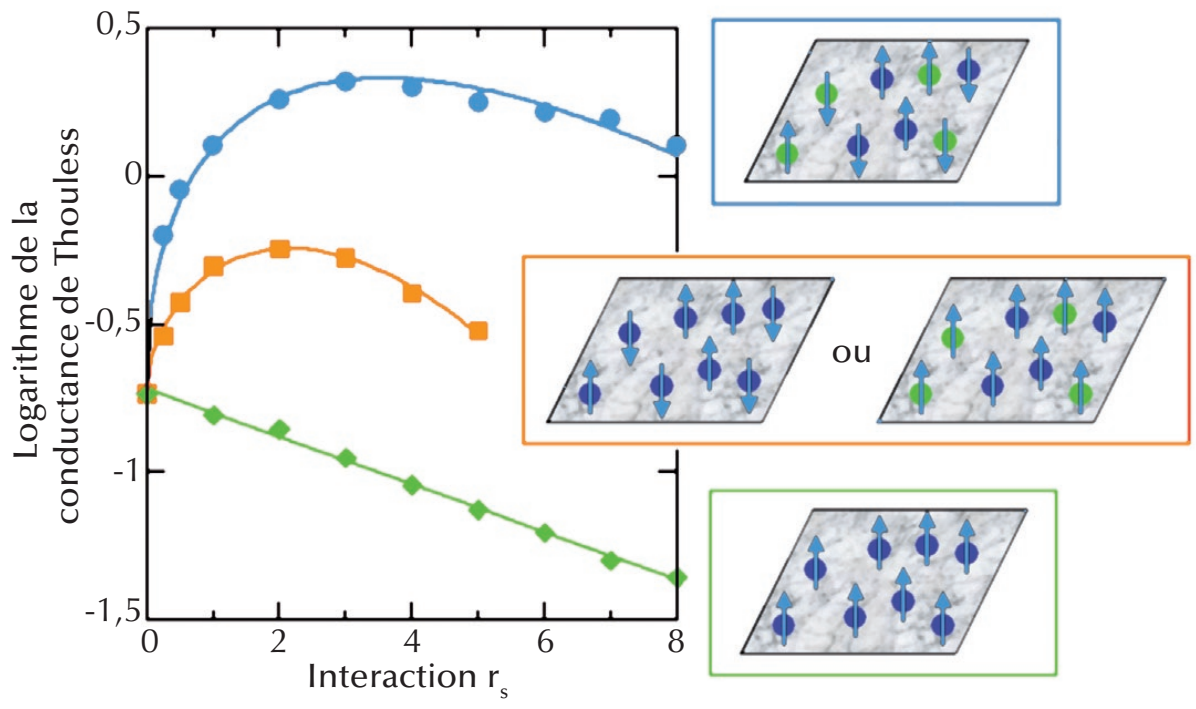

3. Effet des interactions coulombiennes sur la conductance de Thouless d'un système bidimensionnel d'électrons désordonné (de désordre et taille fixés), d'après nos données numériques. Selon la polarisation en spin (up ou down) et en vallée (bleue ou verte) des électrons, les interactions sont soit favorables (carrés rouges et cercles bleus), soit défavorables (losanges verts) au transport.

\section{Localisation d'Anderson et interactions électroniques}

Nos résultats, illustrés par la figure 3, montrent que l'effet des interactions coulombiennes sur la localisation d'Anderson dépend fortement du spin des électrons et du nombre de vallées qu'ils occupent [6], comme le suggèrent les expériences discutées plus haut. Lorsque tous les électrons sont polarisés en spin et en vallée (tous up et bleus par exemple), les interactions font décroître la conductance de Thouless du système (diamants verts) et sont donc nuisibles au transport. À l'inverse, si l'on ajoute un degré de liberté interne, la conductance de Thouless se met à croître lorsque les interactions sont branchées (carrés rouges) et si l'on en ajoute encore un, l'augmentation de conductance est encore plus marquée (cercles bleus). Les interactions aident alors grandement au transport électronique.

Dans une vision effective à un corps (Hartree-Fock), ces résultats peuvent qualitativement s'expliquer par une compétition entre deux effets opposés des interactions. Le premier effet, assez intuitif, facilite la conduction électronique en écrantant le désordre : les électrons, placés de préférence aux puits du potentiel désordonné, induisent un potentiel coulombien moyen qui «bouche» les puits, si bien que le potentiel désordonné effectif s'en retrouve lissé. L'effet est d'autant plus efficace que les électrons ont un nombre élevé de degrés de liberté internes car, en vertu du principe de Pauli, ils peuvent alors peupler en plus grand nombre les puits de potentiel. Le second effet, à l'inverse, renforce la localisation des électrons et cela, indépendamment du nombre de degrés de liberté internes électroniques. Son origine, plus complexe, est liée à la nécessité d'antisymétriser la fonction d'onde des électrons. Lorsque tous ceux-ci sont polarisés en spin et en vallée, ce second terme l'emporte sur le premier (diamants verts), mais lorsque l'on ajoute un degré de liberté interne, la situation s'inverse (carrés rouges puis cercles bleus).

\section{Origine des comportements métalliques expérimentaux}

À partir de notre modèle très simple et de notre méthode numérique, nous avons finalement réussi à proposer un mécanisme qui rend bien compte des comportements métalliques expérimentaux. Son énoncé précis, explicité et justifié ailleurs [8], dépasse le cadre de cet article ; mais l'idée sous-jacente est déjà essentiellement suggérée par l'effet drastique de délocalisation par les interactions, que nous avons observé numériquement dans les systèmes bidimensionnels d'électrons non polarisés en spin 
Expérimental
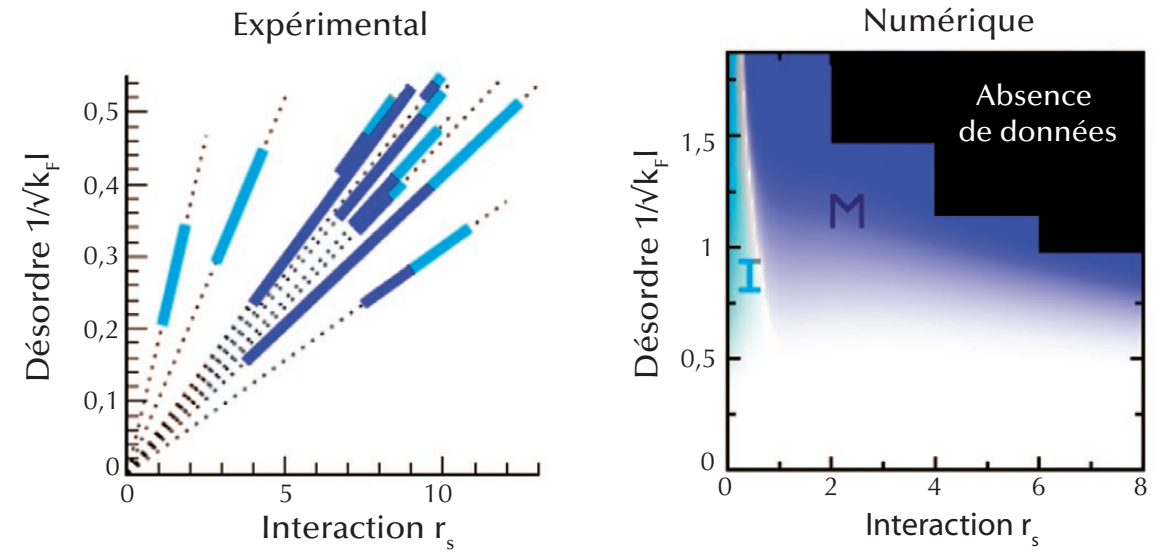

4. Comparaison des diagrammes de phase désordre/interaction obtenus expérimentalement (à gauche) et numériquement (à droite), pour le système bidimensionnel d'électrons formé dans un Si-MOSFET. Les unités des axes des ordonnées et des abscisses sont respectivement les paramètres sans dimension de désordre $\left(k_{\mathrm{F}} \mathrm{I}^{-1 / 2}\right.$ et d'interaction $r_{s}\left(r_{s}\right.$ défini dans le texte, $k_{F}$ vecteur d'onde des électrons au niveau de Fermi et I libre parcours moyen d'un électron entre deux chocs élastiques, qui diminue quand le désordre augmente). Les régions en bleu clair (où se situent les expériences des années 80) correspondent à une phase isolante et les régions en bleu foncé (où se situent les expériences de Kravchenko 1994) à un métal apparent. Dans le diagramme expérimental, à chaque échantillon de la littérature correspond une droite, sur laquelle on se déplace en variant la densité d’électrons (en modifiant la tension de grille). Les paramètres d'interaction et de désordre diminuent quand on augmente la densité électronique. Le caractère métallique ou isolant est donné par la dépendance en température de la résistivité du système.

\section{Désordre versus interactions dans l'affaire du transport d'électrons}

Tant que les électrons peuvent être vus comme de petites billes, la résistivité électrique d'un métal s'explique correctement par la présence d'impuretés (et, à température finie, de vibrations atomiques) déviant les électrons de leur course à travers le réseau d'atomes : plus les électrons sont " ballottés », plus la résistivité est élevée, mais ils finissent toujours par passer.

À très basse température, cette vision simpliste n'est plus valable, car les électrons se comportent alors aussi comme des ondes. Réfléchies par le potentiel de désordre dû aux impuretés, ces ondes peuvent interférer de manière destructrice de sorte que, finalement, les électrons se retrouvent localisés dans une région finie de l'espace. L'effet est particulièrement fort à une et deux dimensions où un petit désordre, même infinitésimal, est suffisant à la localisation (alors qu'en dimension trois, un désordre plus élevé est nécessaire). Ce phénomène quantique, connu sous le nom de localisation d'Anderson [1], explique comment le désordre peut empêcher la propagation du courant dans un matériau, en bloquant littéralement les électrons. On doit sa découverte à Philip Anderson, qui le suggéra en 1958 et obtint pour cela le prix Nobel en 1977.

Mais I'histoire ne s'arrête pas là ! En effet, les interactions coulombiennes, qui repoussent les électrons (chargés négativement) les uns des autres, peuvent venir perturber l'action du désordre en brouillant la cohérence de phase des ondes, absolument nécessaire aux interférences dans le processus de localisation d'Anderson. Désordre et interaction sont ainsi intimement mêlés lorsqu'il s'agit de discuter du transport électronique à basse température. En pratique, l'importance des interactions dans le système - caractérisée par le paramètre $r_{s}$, rapport de l'énergie potentielle de Coulomb sur l'énergie cinétique des électrons - peut être modifiée expérimentalement en variant la densité électronique. Lorsque celle-ci est diminuée, le paramètre $r_{\mathrm{s}}$ augmente (car si l'énergie coulombienne diminue, l'énergie cinétique diminue encore plus vite). Ce faisant, c'est aussi le désordre effectif vu par les électrons qui est modifié, même si la quantité d'impuretés dans le matériau est fixée au moment de son élaboration. et en vallée (cercles bleus de la figure 3). Sur la figure 4, nous comparons pour les Si-MOSFETs le diagramme de phase désordre/interaction prédit numériquement d'après ce mécanisme, à celui obtenu expérimentalement. Manifestement, l'accord entre ces deux diagrammes est plus que bon, d'autant qu'il a été obtenu sans paramètre ajustable. En particulier, nous prédisons des comportements métalliques au centre du diagramme, à désordre et interaction intermédiaires, exactement là où des comportements métalliques ont été observés expérimentalement. À faible interaction, nous trouvons, à l'inverse, des comportements isolants, en accord avec les expériences. $\mathrm{La}$ région isolante, à fort désordre et forte interaction, échappe en revanche à notre description, en l'absence de données de calcul dans ce régime extrême.

\section{Le comportement à $0 \mathrm{~K}$}

Les résultats présentés ci-dessus ne permettent toutefois pas de conclure à la stabilisation d'un vrai métal par les interactions, au sens strict de température nulle. En effet, pour traiter proprement le problème théorique de température nulle, il est nécessaire d'extrapoler nos résultats à la limite thermodynamique, c'est-à-dire à un système de taille infinie ${ }^{(4)}$. Or, une analyse poussée de nos données en théorie d'échelle montre que la conductance de Thouless du système décroît inévitablement lorsque celui-ci est agrandi, que l'on prenne en compte ou non les interactions entre électrons. Ainsi, même si la conductance du système est augmentée par les interactions, elle finit toujours par s'annuler à la limite thermodynamique, si bien qu'à température nulle le système reste en fait rigoureusement isolant.

Soulignons-le, cette conclusion selon laquelle il ne peut exister de métal en dimension deux n'est pas contradictoire avec les expériences où des comportements métalliques ont été observés. En effet, dans ces expériences, ce n'est pas la limite théorique de température nulle qui est explorée, mais une physique de beaucoup plus haute énergie, qui implique les états excités du système [7].

On comprend alors que l'effet de délocalisation par les interactions puisse suffire à l'observation d'un métal apparent à ces température expérimentales "élevées ", bien qu'il soit insuffisant pour stabiliser un vrai métal à $0 \mathrm{~K}$. 


\section{Bibliographie}

1 - A. Lagendijk et al., "Fifty years of Anderson localization", Physics Today 62 (2009) 24

2 - P. A. Lee et T. V. Ramakrishnan, "Disordered electronic systems”, Rev. Mod. Phys. 57 (1985) 287.

3 • S. V. Kravchenko et al., Phys. Rev. B 50 (1994) 8039.

4 - E. Abrahams et al., Rev. Mod. Phys. 73 (2001) 251.

$5-G$. Fleury et X. Waintal, "A numerical finite size scaling approach to many-body localization”, Phys. Rev. Lett. 100 (2008) 076602

6 - G. Fleury et X. Waintal, "Many-body localization study in low-density electron gases: Do metals exist in two dimensions?" Phys. Rev. Lett. 101 (2008) 226803.

7 • G. Brunthaler et al., Phys. Rev. Lett. 87 (2001) 096802.

8 - G. Fleury et X. Waintal, "Energy scale behind the metallic behaviors in low-density Si-MOSFETs". Phys. Rev. B 81 (2010) 165117.

9 • S. V. Kravchenko et al., Phys. Rev. B 51 (1995) 7038.

\section{>>}

\section{Conclusion}

À la question de l'existence de métaux à deux dimensions, nous apportons finalement une réponse nuancée. Nous affirmons qu'il ne peut exister d'état métallique en dimension deux au sens théorique de température nulle, en accord avec le paradigme d'Anderson ; ceci signifie que si l'on était capable d'abaisser davantage la température dans les expériences, la résistivité devrait finalement diverger. Toutefois, nous attribuons les comportements métalliques observés expérimentalement à température finie, non pas à un artefact expérimental ou à un ingrédient ad hoc, mais à un effet intrinsèque des interactions électronélectron. En délocalisant la fonction d'onde électronique, ces interactions favorisent le transport d'électrons, sans pour autant stabiliser une vraie phase métallique. I
(1) L'idée de cette théorie d'échelle est d'étudier la conductance du système lorsque l'on varie la taille de celui-ci. Il est ainsi possible de déduire les propriétés de conduction du système de taille infinie.

(2) Cette méthode permet de résoudre l'équation de Schrödinger à température nulle dans l'espace bidimensionnel (discrétisé et de taille finie, pour les besoins du numérique). Nous avons ainsi étudié des systèmes contenant typiquement une trentaine d'électrons dans un millier de sites. Les paramètres physiques réalistes du silicium ont été utilisés comme paramètres d'entrée.

(3) Lorsque le système bidimensionnel est replié sur un tore et qu'un flux magnétique le traverse en son centre, un courant d'électrons apparaît dans le système. La conductance de Thouless est le coefficient de proportionnalité qui relie le courant au flux qui lui a donné naissance (à bas flux).

(4) Cela se comprend en remarquant que plus la température est basse dans le système, plus les ondes électroniques gardent leur capacité d'interférer entre elles sur une grande distance. C'est à cette distance que correspond la longueur de notre système dans nos simulations numériques. 